\title{
La actividad creadora de la causa trascendente
}

Desde siempre, el hombre se ha interrogado sobre la causa y el origen del universo que habita intentando esclarecer el misterio de sus comienzos. Esta pregunta sobre el origen de las cosas, nunca fue una cuestión meramente académica, encaminada solamente a satisfacer nuestra curiosidad intelectual, sino que en su respuesta el hombre espera encontrar los fundamentos de sus normas de comportamiento y el sentido de lo que del futuro puede esperar.

Mitologías y sistemas filosóficos se habian ocupado de esta fundamental cuestión y habian propuesto las más diversas soluciones. La concepción creacionista del pueblo judío, introducida en el mundo de la cultura occidental a través del cristianismo, significó, en este campo, una radical innovación. La idea de que el origen del universo hay que buscarlo en el acto creador de una Causa Trascendente, revoluciona profundamente los esquemas ideológicos greco-romanos y se convierte en cuestión ineludible para todos los pensadores posteriores. Whitehead considera a Aristóteles como el último gran metafísico de Occidente que pudo hablar de Dios $\mathrm{y}$ construir un sistema del mundo, sin prejuicios.

\section{El problema del "cómo" de la creación}

Cuantos en el mundo se profesan cristianos admiten la doctrina creacionista como solución al problema del origen del mundo. Ahora bien, si la admisión del "hecho" de la creación no ha sido nunca problema para quienes dan fe a la revelación que se lo propone, sí lo ha sido, últimamente, la búsqueda de una representación conceptual coherente del "realizarse" de dicho "hecho". La admisión fiducial del hecho de la creación no puede satisfacer plenamente al espiritu, si este hecho nos es presentado en esquemas ideológicos que parecen contradecir a la imagen del mundo que las modernas ciencias positivas y cosmogonías nos presentan. La inquietud que ha surgido entre los creyentes no se dirige al "hecho" 
de la creación, sino que pretende buscar una nueva representación conceptual de la misma.

En este trabajo queremos ocuparnos de una serie de cuestiones relativas a este problema, intentando esclarecer en qué consiste y proponiendo la solución al mismo de dos grandes espíritus, S. Agustín y Teilhard de Chardin. Las ideas de ambos pueden ser una valiosa aportación a la solución de tan importante cuestión.

Pero veamos, ante todo, cómo y porqué surgió la necesidad de nuevas maneras de representarse la actividad creadora.

\section{El actuar creador en un mundo "fijista"}

Un mundo fijista es aquel en el que no existe el movimiento categorial. En un tal universo no se dan cambios ontológicos más que a nivel individual: lo que surge, no son más que nuevos representantes de categorias que existieron inmutables desde el principio. Se trata de un universo definitivamente hecho y acabado desde sus comienzos y lo que en torno a nosotros contemplamos es, fundamentalmente, lo que, en el principio, surgió de las manos de su creador. La causa trascendente actuó creadoramente en el comienzo haciendo venir a la existencia, una vez por todas, cuantas formas de ser lo componen. Solamente la conservación es necesaria para explicar la marcha ulterior de lo creado. Esta posición era la aceptada, no solamente por los teólogos, sino incluso por los más eminentes naturalistas, antes de la aparición de las ideas transformistas ${ }^{1}$.

La polémica del transformismo: el "intervencionismo".

Ahora bien, si durante centurias la humanidad pudo pensar que nuestro universo era un sistema definitivamente "acabado" desde sus comienzos, esta imagen comenzó a cambiar radicalmente al hacer su aparición las primeras ideas transformistas. Estas teorías tuvieron, en un principio, una aplicación bastante restringida pues se limitaban únicamente a la esfera de lo viviente. Pero, a pesar de todo, el mundo comenzó a tener una historia. La Paleontología demostró que no todo habia existido desde el principio, sino que la fauna de cada era geológica poseía peculiaridades propias y desco-

1. Baste recordar el tan traído y llevado texto de Linneo (1707-1778), en el comienzo de su Sistemática: "Tot sunt genera et species quot ab initio creata sunt". Citado en A. MUELLER, Das naturphilosophische Werk Teilhard de Chardins (Freiburg 1964) 32. 
nocidas en otras épocas. Además, entre las distintas faunas parecía existir una relación de deriva. Al parecer, la rica variedad de las especies animales, había sido el fruto de la transformación de unas especies en otras. $\mathrm{Y}$ ello bastaba para poner en entredicho la representación tradicional del actuar creador.

El uso exclusivamente ateo que se pretendió hacer de estas teorías no vino sino a complicar las cosas. La posibilidad de colocar en una cadena generacional las distintas especies animales, incluido el hombre, pareció un argumento suficiente para negar la singularidad y trascendencia de este último y para considerar superflua la existencia de una causa creadora: si en el cosmos todo tiene antecedentes causales y no hay una diferencia esencial entre los estratos que lo componen, todo tiene una causa adecuada en sus antecedentes y para nada se necesita una causa extracósmica. En lugar de confinarse en los límites metodológicos de sus disciplinas, los científicos se dedicaron a "filosofar", deduciendo conclusiones metafísicas de premisas de orden biológico ${ }^{2}$. Determinadas corrientes filosóficas utilizaron, además, sin muchos escrúpulos, las nuevas ideas como instrumento ideológico de su ateísmo ${ }^{3}$. No es, pues, de extranar que todas las ideologías creacionistas mirasen con recelo las teorias transformistas, visto el uso que de ellas se hacia.

Las reacciones fueron muy variadas. Hubo quien, con muy poco éxito, se dedicó a hacer "ciencia" para, mostrando las lagunas y dificultades de las teorías transformistas, minar su base científica y continuar manteniendo su mundo fijista. La mayor parte de los teólogos, sin embargo, se plegó, con el tiempo, a la fuerza de los hechos e intentó "completar", en conformidad con las circunstancias, la representación tradicional del actuar creador. Surgió así la teoría que podemos llamar "intervencionista". Manteniendo intacta la concepción tradicional de una creación inicial, eran postuladas sucesivas intervenciones creadoras cada vez que algo nuevo hacía su aparición, para añadirse al conjunto de lo ya existente.

Este postular la intervención de la causa creadora para cada nueva aparición, era consecuencia de toda una serie de presupues-

2. Darwin, por ejemplo, se expresaba en los siguientes términos: "Nevertheless the difference in mind between man and the higher animals, great as it is, certainly is one of degree and not of kind": Descent of man (London 1899) 126.

3. Engels escribía a Marx en 1859: "Darwin... ganz famos. Die Theologie war noch nicht kaputt gemacht. Das ist jetzt geschehen". Marx responde a la misiva de Engels, diciendo que la obra de Darwin: "...die naturhistorische Grundlage für unsere Arbeit enthält". 
tos metafísicos implícitos. Por una parte, se intentaba mantener a toda costa, la inmutabilidad y mutua irreductibilidad de las especies. Además se queria para las nuevas apariciones una causa explicativa adecuada. $Y$ partiendo del supuesto de la superioridad de cada nueva aparición sobre lo que la había precedido e identificando superioridad biológica con superioridad metafísica absoluta, era necesario el recurso a la causa trascendente, cada vez que una nueva especie hiciese su aparición. $Y$ esto por la sencilla razón de que lo más no puede venir de lo menos. El recusar a las causas segundas toda participación causal en la aparición de lo nuevo se justificaba diciendo que la creación era de competencia exclusivamente divina. En el fondo, había un temor inconsciente de disminuir la grandeza del creador si se hacía participar a las creaturas en la aparición de seres nuevos, irreductibles a los anteriores.

En contra de lo que muchos creen, esta posición no fue defendida exclusivamente por teólogos, sino que eminentes naturalistas también la hicieron suya. Recuérdese, por ejemplo, cómo G. Cuvier (1769-1832) defendió victoriosamente en 1830, ante la Academia parisina y contra Geoffroy St. Hilaire (1772-1844) la constancia e inmutabilidad de las especies. $Y$ ello a pesar de los descubrimientos de la Paleontología. Para resolver el problema de las diferencias entre las faunas fósiles de las distintas eras geológicas, recurrió a la teoría de las "catástrofes sucesivas": al término de cada era geológica una catástrofe de amplitud cósmica hubiese destruido la fauna existente y un acto creador hubiese llamado a la existencia a una nueva fauna. Así se explicarian las diferencias que la Paleontologia encuentra entre los restos fósiles de las distintas eras geológicas.

Estas representaciones "intervencionistas" de la actividad crea-

4. A nosotros nos parece que esta doctrina de la inmutabilidad de las especies no es un elemento semítico en la doctrina creacionista, sino una intrusión del platonismo en los esquemas cristianos. Según Platón, de cada esencia existe un modelo eterno e inmutable, identificado con una Idea subsistente. Crear, para Platón, consiste en que el Demiurgo copie en la "causa errante" (la materia) los modelos eternos. En el cristianismo no desaparece totalmente el mundo de las ideas de Platón, sino que es colocado y unificado en el mundo inteligible del Verbo. Pero aunque las ideas dejen de ser substancias independientes, no por eso dejan de ser eternas e inmutables y de servir de modelo a la creación. Por eso el mundo es copia y huella de Dios en todos los autores cristianos. La creación es una imitación en el ser contigente y material de los modelos eternos. En un tal esquema, cada esencia realmente existente es un reflejo de un modelo eterno y de una "species metaphysica" inmutable. Consiguientemente es absolutamente impensable que unas esencias puedan originarse evolutivamente de otras, puesto que todas ellas existen ya independientes en la eternidad. 
dora nos parecen artificiosas y poco satisfactorias para el espiritu, siendo, por ende, innecesarias. Si su aparición es explicable, como reacción a un medio ideológico hostil y ateo, el número de dificultades de toda índole con que topa, hacen muy difícil, por no decir imposible, su aceptación.

El intervencionismo nos presenta, en primer lugar, un esquema bastante antropomórfico del actuar creador, calcado en el modelo de una causalidad eficiente de tipo artesanal: una especie de "Deus ex machina" que concluye su obra, mediante una serie de sucesivos y periódicos retoques. La misma terminologia (intervención), parece indicar sucesividad en la actividad causativa creadora. Lo cual, como bien vio S. Agustín, es inadmisible: el actuar creador es actividad de un Acto Puro y, como tal, se pone una sola vez en la eternidad. Dios es creador una vez por todas y aunque se admita una sucesión en el aparecer de los efectos, es necesario mantenar la unidad y simultaneidad en el ejercicio de la acción causadora creativa ${ }^{5}$.

Otra dificultad, no pequeña, sería el determinar cuándo debe tener lugar la intervención creadora. Es sumamente difícil, desde el punto de vista filosófico, el poder afirmar que en lo recién aparecido se da un aumento absoluto de densidad ontológica que supera metafísicamente a sus antecedentes ${ }^{6}$.

Esta teoria, finalmente, no respeta totalmente las apariencias que de la historia del mundo nos propone la ciencia: no se trata solamente de explicar un aparecer sucesivo de distintas formas de ser, sino de la concatenación y ligazón orgánica que entre ellas existe. La historia de la vida no habla solamente de sucesión, sino también de deriva. Ahora bien, la intervención es concebida como creación estricta, es decir, como posición de radical novedad, donde lo nuevo que aparece no se deriva, en ningún sentido, de lo anterior, sino que se yuxtapone a ello. Responder que la indudable continuidad monfológica entre las especies y su ligazón orgánica no responden ni son signos de una deriva objetiva, sino que son aparentes y obedecen a un plan divino, para nosotros incomprensible, nos parece un intento poco serio de querer mantener, por 320.

5. De Genesi ad litt. IV, 33, 51: PL 34, 317-318; IV, 34, 53-55: PL 34, 319-

6. Solamente con relación a la aparición del alma humana, postula la Iglesia católica un especial concurso del Dios creador. Concurso que, por lo demás, no debe ser entendido necesariamente como una intervención independiente de toda la evolución cósmica anterior. 
encima de todo, la inmutabilidad e incomunicabilidad de las especies animales?.

La imagen del universo en la ciencia actual.

Desde la polémica del transformismo ya ha pasado más de una centuria. Ello ha hecho que ciertas posturas y actitudes emocionales hayan cedido ante la fuerza de los hechos y ante la evidencia de que una interpretación "creacionista" de los mismos es posible.

Hoy no se puede negar la validez y universal aceptación de las teorías eivolucionistas como inistrumento teórico descriptivo de la historia del mundo. Si durante siglos la humanidad pudo pensar que el mundo en que vivía era un sistema definitivamente acabado, hoy ha tomado conciencia de que esta errónea representación era debida a una falta de perspectiva.

Los límites del universo, tanto en el espacio como en el tiempo, han reculado hasta lugares insospechados. El movimiento y la historicidad, que en el siglo pasado se usaban solamente para describir la biosfera, han invadido la totalidad de los estratos cósmicos. La evolución es una ley universal, desde los más primitivos y rudimentarios movimientos galácticos hasta los animales biológicamente más perfectos. En este marco ideológico ampliado, el transformismo biológico no es más que una etapa en el proceso general.

Todo tiene una historia y todo ha colaborado, de alguna manera, a la aparición de lo que viene detrás. Las "esencias" han dejado de ser bloques aislados para convertirse en etapas de un proceso. Cada ser se prolonga indefinidamente en la noche de los tiempos, siendo todo el universo que lo precede su matriz.

Pero, si esto es así ¿cómo pudo el hombre estar tan engañado durante tanto tiempo? ¿cómo no fue capaz de tomar conciencia del movimiento en que se encuentra inmerso? ¿por qué el universo ha dejado siempre al hombre una irresistible sensación de fijeza y estabilidad? ¿por qué lo ha considerado siempre el marco fijo en que se desenvuelven las existencias individuales? Sencillamente porque no disponía de la perspectiva suficiente para poder detectar el movimiento. Los movimientos cósmicos, la evolución, proceden a un ritmo tan increíblemente lento en comparación de la vida, no ya sólo de cada hombre, sino incluso de toda la humanidad, que era

7. Así pensaba el gran naturalista suizo, nacionalizado norteamericano, Louis Agassiz (1807-1873), que postulaba nada menos que veinte "intervenciones", solamente para explicar la diversidad de las razas humanas. 
prácticamente imposible captar la sucesión y el movimiento. Es sintomático el que cuando las ciencias han puesto a disposición del hombre una capa de tiempo lo suficientemente amplia, es cuando éste ha empezado a tomar conciencia del carácter dinámico del universo que habita. De hecho, solamente a raíz del desarrollo de las ciencias paleontológicas que ponen a nuestra disposición, en sus fósiles, millones de años de la historia de la vida, se ha percatado el hombre del carácter ilusorio de la concepción fijista del universo $^{8}$.

Teoría agustiniana de las razones seminales.

Desde el momento que las teorias transformistas dejaron de ser miradas por los teólogos como absolutamente incompatibles con el dogma de la creación, se ha intentado repetidamente hacer de $\mathbf{s}$. Agustín un precursor ideológico del evolucionismo. El sería la famosa excepción que confirma la regla en el horizonte intelectual de la teología católica, dominado, durante siglos, por los esquemas fijistas.

Nosotros, sin embargo, creemos que hay que matizar bastante esta afirmación, ya que si es indudable que la teoria agústiniana de las razones seminales difiere profundamente de los esquemas normales de la teología católica y es, además, compatible con algunos aspectos de la moderna imagen del mundo, no puede, en rigor, ser considerada como evolucionista, ni en su intención, ni en la materialidad de su contenido. Pero expongamos, ante todo, brevemente, en qué consiste.

El texto principal en que s. Agustin expone su teoria es el comentario a los primeros capitulos del Génesis ${ }^{9}$ y su finalidad no es

8. Una nueva ciencia que puede ampliar también nuestros conocimientos de pasadas épocas de la evolución cósmica es la astrofísica y, más en concreto, la espectrografía. El análisis de la luz filtrada y descompuesta nos proporciona datos valiosísimos sobre la temperatura, composición física y química, velocidad y posición del cuerpo que la emite. Ahora bien, muchos de estos cuerpos emisores de luz se encuentran a distancias increíbles de la tierra (ciertos quasars distan de nosotros, al parecer, ocho mil millones de años de luz!). Esto quiere decir, que la luz que nuestros científicos descomponen y analizan, ha viajado por el espacio durante millones de años antes de llegar a sus telescopios y que lo que indican por consiguiente, no es el estado actual del cuerpo emisor de luz, sino su estado hace millones de años. Esto quiere decir que esa luz es, en cierto sentido, un "fósil", en cuanto que nos proporciona datos sobre la materia, tal como ésta era hace millones de años. De esta manera podemos estudiar la evolución del cosmos desde épocas remotísimas a la nuestra. Aunque el cuerpo emisor de luz haya cambiado o desaparecido, nos ha dejado, viajando por el espacio, un mensaje luminoso en el que nos cuenta su vida pasada.

9. De Genesi ad litt.: PL 34, 246-486. 
ni siquiera filosófica, ya que lo que en realidad trata de resolver con ella, es un problema de orden exegético.

Al tratar de interpretar el texto genesiaco de la creación se topa con lo que él considera una contradicción. $Y$ es que la naturaleza de la causa creadora, acto puro en el que no puede existir la sucesión ${ }^{10}$ y otros textos de la misma Escritura ${ }^{11}$, exigen la ausencia de temporalidad en el acto creador, es decir, que la creación sea instantánea. $\mathrm{Y}$, sin embargo, el Génesis, en su relato, nos habla de una creación temporal y sucesiva.

Para solucionar este problema propone S. Agustín su teoría de

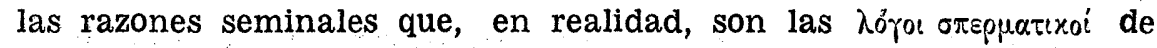
los estoicos. Dios habría creado todo al mismo tiempo, pero no del mismo modo. Ciertas formas de ser hubiesen surgido perfectas y acabadas de las manos del Creador ${ }^{12}$, mientras que otras 10 hicieron sólo virtual e incoactivamente ${ }^{13}$. De estas últimas fueron creadas sus "razones seminales", que son una especie de virtualidades o principios de desarrollo, ínsitas desde el primer momento en el universo y que, bajo el influjo de la divina conservación, darán lugar a formas de ser acabadas y completas con el correr del tiempo. Ahora bien, de cada forma de ser existe una razón seminal propia y distinta. Consiguientemente el movimiento óntico a que las razones seminales dan lugar no es un movimiento evolutivo de deriva; actual o incoativamente, todo existe desde el principio $\mathrm{y}$ no hay comunicación orgánica entre las diversas formas de ser por lo que a su originarse se refiere.

Con estos elementos es bastante difícil querer hacer de $\mathbf{S}$. Agustín un evolucionista. No lo es en la intención, pues el contexto y las motivaciones de sus teorias no son observaciones de tipo científico ni reflexiones de orden cosmológico, sino simples consideraciones de interpretación exegética: se trata de resolver un problema planteado por la Biblia recurriendo a una conocida teoría fllosófica de la época.

Además, hablando estrictamente, no hay aparición de nada nue-

10. De Genesi ad litt. IV, 33: PL 34, 317; IV 34, 53-55: PL 34, 319320.

11. S. Agustín se refiere al conocido texto de Eccl. 18,1: "Deus creavit omnia simul", interpretando "simul" en sentido temporal. En la exégesis moderna este "simul" tiene un sentido de totalidad: Dios creó todo sin excepción (Cf. Biblia de Jerusalén).

12. S. Agustín enumera los ángeles, el firmamento, el mar, las estrellas...: De Genesi ad litt. IV 1, 2: PL 34, 339.

13. "Invisibiliter, potentialiter, quomodo fiunt futura non facta": De Genesi ad litt. VI, 6, 10: PL 34, 343. 
vo, en cuanto que, aunque fuese germinalmente, todo existió desde el principio. $Y$ tantas formas de ser como razones seminales existieron desde el principio. Desde este punto de vista $\mathbf{S}$. Agustín puede ser considerado un fijista que piensa la creación según el esquema platónico de los modelos eternos a que anteriormente aludimos. Los modelos eternos de Platón, convertidos, en el cristianismo, en el mundo inteligible del Verbo, continúan ejerciendo la misma función. De cada forma de ser existe, desde siempre, un modelo eterno e inmutable, realizado en la materia mediante un acto creador. El que la realización o manifestación de esa realización tenga lugar en distintos momentos para nada influye en el carácter inmutable de los modelos eternos. Ahora bien, evolución no significa solamente sucesiva aparición en el tiempo, sino deriva y transformación de unas formas de ser en otras.

$\mathbf{Y}$, sin embargo, tampoco puede ser denominada fijista, en sentido estricto, la teoría de las razones seminales, en cuanto que en el fijismo todo aparece acabado desde el principio, mientras que en la teoria agustiniana, aún habiendo sido creado todo en los comienzos, hay sucesión a la hora de manifestarse los seres en cuanto acabados. Consiguientemente, parece conveniente proponer un nuevo término para designar esta especie de fijismo. $Y$ el más adecuado, a nuestro entender, es el de sucesionismo. Querer denominar evolucionista a la teoría agustiniana es ignorar el significado corriente de este término: en la teoría de S. Agustín no hay posibilidad de que una forma de ser intervenga en la aparición de otra, en cuanto que la existencia actual o virtual de todo remonta al comienzo de los tiempos.

Esta teoria, finalmente, no responde plenamente a la descripción que las ciencias positivas nos dan de la historia del mundo. Tales ciencias, en efecto, no solamente constatan una aparición sucesiva de los estratos ónticos, sino que detectan, además, una ligazón orgánica entre los mismos y una dependencia causal, de la naturaleza que sea entre lo posteriormente aparecido y sus antecedentes. Naturalmente, esto no quiere decir necesariamente que los antecedentes, aún colaborando, sean la causa única y adecuada de la aparición de los efectos.

\section{La concepción teilhardiana del actuar creador}

Es difícil el estudio de una doctrina particular en Teilhard de Chardin en cuanto que en él, quizás más que en otros autores, el contexto general tiene una importancia fundamental a la hora de 
valorar doctrinas concretas. Existe una gran unidad entre los elementos de su sistema y pretender estudiar uno de ellos fuera del todo nos hace correr el peligro de parcialidad a la hora de juzgar. Dados, sin embargo, los límites materiales de este trabajo, no tenemos más remedio que prescindir de una exposición global del sistema, dando por supuesto, por parte del lector, el conocimiento de sus grandes líneas.

Digamos, ante todo, que muchas de las ideas teilhardianas en torno a la creación son, no sólo comunmente aceptadas por los teólogos católicos, sino que las repiten en sus manuales sin tomarse la molestia de citar al autor de lo que, al parecer, se ha convertido en patrimonio común. $\mathrm{Y}$ sin embargo, tales ideas fueron, hace un decenio, causa de múltiples tragedias personales para su autor.

Es evidente que no todas sus doctrinas tienen el mismo valor y que no todas son igualmente utilizables. Nosotros comenzaremos por exponer sus ideas para criticarlas a continuación, mostrando qué elementos son menos convincentes o inaceptables para el dogma.

En qué consiste fundamentalmente el "ser creado".

Si queremos representarnos de alguna manera la actividad creadora, es necesario que determinemos, ante todo, qué significa la expresión "ser creado".

Teilhard comienza con una definición negativa, rechazando la representación tradicional, basada exclusivamente en un modelo de causalidad eficiente de tipo artesanal ${ }^{14}$. "Ser creado" significa para él, positivamente, una indicación del estatuto óntico de lo contigente. La creación consiste, esencialmente, en una fundamental dependencia con relación al ser necesario, por la cual, todo lo que no sea El, es secundario y participado ${ }^{15}$. Lo que constituye el "ser creatura" es el no poder existir sino dependiendo del Ser Absoluto. "Ser creado" significa, consiguientemente, no tanto el ser fruto de una actividad causal eficiente, como en existir de tal manera que haya

14. Au début, la position extra nihilum d'une certaine somme de puissances (phase créatrice initiale). Après cela, un développement autonome de ces puissances, entretenues par la conservation (phase de transformation par les causes secondes). Ensuite, de nouvelles positions extra nihilum, chaque fois que le développement historique du monde nous révèle des accroissements vrais: apparition de la vie, d'une espèce métaphysique, de chaque âme humaine...": Sur la notion de transformation créatrice: (Oeu. X, 29-30). Citaremos a Teilhard según las obras completas que indicamos en la Bibliografía. Si alguna vez traducimos algún texto, la traducción será nuestra.

15. Les fondements et le fond de l'idée d'évolution: (Oeu. III, 188). 
que buscar en otra cosa el fundamento del propio existir. La representación concreta de este modo de ser-en-dependencia es secundaria frente al hecho fundamental.

Una primera consecuencia de esta doctrina es la necesidad de concebir la creación como incesante e ininterrumpiaa relación entre la creatura y el Creador. Si "ser creado" consiste en que la propia existencia, hasta en sus últimas fibras, depende de otra instancia distinta del propio ser, esta relación de dependencia nunca está interrumpida mientras existan cosas. La creación es coextensiva a la totalidad temporal-espacial del universo. Dios es creador en todo instante y el mundo continúa siendo creado en cada momento. Consiguientemente la creación no es solamente un acto instantáneo e irrepetible en el origen de los tiempos, sino presencia continua y vivificante de lo Necesario en cada punto de lo contingente ${ }^{16}$.

El acto creador no es, pues, una actividad episódica, ejercida una vez por todas en el comienzo de los tiempos, sino una ininterrumpida donación de ser. Es un gesto continuo, espaciado y coincidente con todos los instantes de la temporalidad. La creación continúa todavía $\mathrm{y}$, en cada uno de sus momentos, el mundo es arrancado a la nada ${ }^{17}$. El acto creador se ejerce sobre todo el universo, tanto en el espacio como en el tiempo ${ }^{18}$.

"No se puede decir que hay un momento en que Dios crea y un tiempo en que actúan las causas segundas. El acto creador es uno y coincide con la actividad de las causas segundas... Comprendida de esta manera, la creación no es una intrusión periódica de la Causa Primera, sino una actividad coextensiva a la duración total del Universo" ${ }^{19}$.

Ahora bien, aunque el término de la actividad creadora sea el universo en su totalidad, no por eso hemos de concluir que la actividad creadora, en sí misma y en cuanto tal, sea temporal. Lo que es temporal es la realización. Dios actúa en la eternidad y en él no hay sucesión. $\mathrm{Y}$ precisamente por ser eterno puede estar presente en cada punto de la temporalidad. Solamente en los efectos hay su-

16. Sur la notion de transformation créatrice: (Oeu. $\mathrm{X}, 31$ ); Christologie et évolution: (Oeu. $\mathrm{X}, 101$ ).

17. Le milieu mystique: (ETG 149). Usamos ETG como abreviatura de Ecrits au temps de la guerre. Paris, 1965.

18. Mon univers (1924): (Oeu. IX, 107). Indicamos la fecha del ensayo, para distinguirlo del que, con el mismo nombre, publicó en 1918: (ETG 263-279).

19. Sur la notion de transformation créatrice: (Oeu. $\mathrm{X}, 31$ ). 
cesión, existiendo simultaneidad en el ejercicio de la acción causativa.

Indetectabilidad empirica del actuar creador

Nada se realiza en nuestro universo que no dependa del Ser Trascendente. El Ser Necesario tiene que estar siempre presente para hacer que lo contingente pueda existir. Ahora bien, esto no quiere decir que la actividad creadora deba ser de tal naturaleza que haya de manifestarse como una "epifania", ruptura o intrusión. Es más, en realidad parece confundirse con la actividad propia de las causas segundas adquiriendo, a veces, la figura de un azar o de una inmanencia inaprensible ${ }^{20}$. Al actuar la Causa Trascendente "...no hay ruptura ni desgarrón, sino que la red de los determinismos continúa virgen y la harmonía de los desarrollos orgánicos se prolonga sin disonancias" ". Con esta tesis se opone Teilhard a quienes quieren representarse la actividad creadora al modo de una intrusión en lo ya existente y, sus efectos, como aparición de algo radicalmente nuevo, que no guardase ninguna relación con to precedente (intervencionismo). El se rebela violentamente contra quienes, queriendo magnificar el poder y la omnipotencia divinas, necesitan ver signos externos y tangibles de este poder, pidiendo para ello una actuación epifánica y arbitraria de la Trascendencia en el sistema de lo creado.

Concausalidad de lo creado en la creación

Aunque el influjo creador de la Causa Trascendente sea la actividad de una causa de "fuera del mundo", nunca se ejerce "sin el mundo", es decir, sin tener en cuenta todo el sistema de lo ya existente. Siempre que algo nuevo va a aparecer, el mundo "es tenido en cuenta" y juega un papel concausal en dicha aparición. Dios es la causa última y esencial, pero su acción creadora se ejerce a través de las causas segundas y usando como punto de partida todo lo ya existente:

"La creación se continúa, en cada momento, en función de todo lo ya existente. Por consiguiente no podemos hablar, en sentido propio, de nihilum subjecti, a no ser que consideremos el universo en su formación total a través de todos los siglos" ${ }^{22}$.

20. Note sur les modes de l'action divine dans l'univers: (Oeu. $\mathrm{x}, 36$ ).

21. Note sur les modes de l'action divine dans l'universe: (Oeu. X., 38 ).

22. Sur la notion de transformation créatrice: (Oeu. $\mathrm{X}, 32$ ). 
Por ello, no es pensable el que Dios cree seres aislados y sin contexto. Cada nueva aparición está preparada y preanunciada por todo 10 anterior ${ }^{23}$.

Una nueva forma de ser surgida no produce la impresión de algo imprevisto, sino que más bien parece el fruto esperado de un proceso de gestación cósmica. Lo que aparece no se yuxtapone simplemente a lo ya existente, sino que es como la coronación de un largo proceso preparatorio y parece dotar de sentido a todo lo anterior. $\mathrm{Y}$ es que el creador utiliza todo lo ya existente cuando ha de llamar una nueva forma de ser a la existencia. Por consiguiente, todo tiene antecedentes y parece hundir sus raices en la totalidad del cosmos precedente ${ }^{24}$. El mundo, en su totalidad, parece ser la matriz universal de cada nuevo ser creado. $Y$ entonces la acción creadora no es "violenta" ni se manifiesta como intrusión detectable; sino que parece coincidir, en cada momento, con el natural desarrollo del universo.

Teilhard utiliza una bella imagen para ilustrar este modo de actuar del creador. Como punto de partida nos propone una representación imaginaria del sistema cósmico total: "Imaginémonos una esfera y, en su interior, un gran número de resortes, apretados los unos contra los otros. Concedamos a estos resortes la facultad de apretarse o de aflojarse libre y espontáneamente. Un tal sistema podría representar el universo y el conjunto de actividades, solidarias unas de otras, que lo componen" ${ }^{25}$. Si intentamos ahora representarnos, mediante un artificio, el obrar de la Causa Creadora en este modelo imaginario del universo, diremos que se ejerce continua y simultáneamente sobre la totalidad de los elementos de la esfera. De tal manera que, para conseguir un efecto preciso en un

23. “...il semble contradictoire (à la nature de l'être participé) d'imaginer Dieu créant une chose isolée... Pour arriver à faire un âme, Dieu n'a donc qu'une seule voie ouverte à sa puissance: créer un monde". Note sur les modes de l'action divine dans l'univers: (Oeu. $\mathrm{X}, 42)$.

"Créer, jusqu'ici, avait été pris comme une opération divine susceptible de revêtir des formes absolument arbitraires. Dieu, admettions nous (au moins implicitement) était libre et capable de faire surgir de l'être participé dans n'importe quel état de perfection et d'association. Il pouvait le placer de plain-pied, à son gré, à un point quelconque entre zéro et l'infini. Ces vues imaginaires me paraissent en désaccord avec les conditions les plus profondes de l'être telles qu'elles se manifestent dans notre expérience": Christologie et évolution: (Oeu. $\mathrm{X}, 101$ ).

24. ...les éléments les plus parfaits du monde se forment successivement au moyen des moins parfaits, à partir des états inférieurs de l'existence". L'union créatrice: (ETG 176).

"Sans un longue période de maturation, aucun changement profond ne peut se produire dans la nature". Le phénomène humain: (Oeu. I, 87).

25. Note sur les modes de l'action divine dans l'univers: (Oeu. $\mathbf{x}, \mathbf{3 5}$ ). 
determinado punto, no necesita intervenir precisamente en ese punto, sino que ejerce su influjo sobre la totalidad del conjunto y de una manera tan sabia que el efecto, además de producirse infaliblemente, parece un producto normal de todo el conjunto. De la misma manera es utilizado el universo para cada nueva creación. Cada nuevo ser surgido procede de las manos de Dios a través de una gestación universal ${ }^{26}$.

\section{Trascendencia e interioridad de la acción divina}

Una nueva característica que hemos de afirmar de la acción divina y en relación directa con su trascendencia, es su interioridad. Dios no obra "desde el exterior" de las cosas, sino que su influjo se hace sentir desde dentro, en lo más íntimo de las naturalezas:

"Cuando la Causa Primera obra, no lo hace intercalándose entre los elementos de este mundo, sino que lo hace directamente, desde el interior de los seres" ${ }^{27}$.

Su acción no comporta una ruptura con las naturalezas individuales, ni una desarmonía con la marcha del conjunto, sino que es elevación y vivificación de la actividad propia de las causas segundas.

Volviendo a su ejemplo de la esfera, habría que añadir un nuevo elemento para describir lo propio de la acción divina: al ser que obraba simultáneamente sobre la totalidad de los elementos de la esfera, lo imaginábamos como exterior a ella. Ahora es necesario que lo imaginemos obrando desde el interior de los mismos resortes, aumentando o disminuyendo su tensión hasta el límite de su elasticidad (actual o posible) y dirigiendo, imperceptible pero infaliblemente, la marcha del conjunto ${ }^{28}$.

Nada más alejado del pensamiento teilhardiano, que una presencia panteística de identificación. Si Dios puede obrar de este modo sobre las naturalezas es en virtud, precisamente, de su trascendencia:

"Incapaz de mezclarse y de confundirse con el ser participado que sostiene y anima, Dios está en el movimiento, el desarrollo y el término de todas las cosas" ${ }^{29}$.

26. Note sur les modes de l'action divine dans l'univers: (Oeu. $\mathrm{X}, 36$ ).

27. Comment se pose aujourd'hui la question du Transformisme: (Oeu: III, 39):

28. Note sur les modes de l'action divine dans l'univers: (Oeu. $\mathbf{X}, 37$ ).

29. La vie cosmique: (ETG 37). 
De todo lo expuesto se deduce una confirmación de la primera afirmación con que comenzamos el análisis relativo a Teilhard de Chardin: la acción divina creadora es empíricamente indetectable, pues parece coincidir, por el modo en que se ejerce, con la marcha "normal" de nuestro universo. Actúa de la misma manera que un alma: presente en todos los puntos del conjunto, es empíricamente ilocalizable en ninguno de ellos:

"Al ejercerse en el seno del mundo, lo propio de la acción divina es, precisamente, el no poder ser detectada ni aquí ni allá..., sino de encontrarse localizada en la totalidad de las actividades segundas que sostiene, finaliza $y$, en cierto modo, eleva" ${ }^{30}$.

Por eso es improcedente hablar de ella en una historia puramente fenomenológica del devenir cósmico: en una descripción de las apariencias de la evolución, la acción creadora no es un fenómeno que se manifieste externamente, sino que, en sí misma, permanece oculta. Solamente los efectos son aprehensibles. Es más, actúa de tal manera, que todos los efectos tienen antecedentes cósmicos. Volvamos de nuevo al ejemplo de la esfera:

"Para los resortes de la esfera, en los cuales se va a ejercer la influencia creadora, el impulso viene de todas las partes a la vez. $Y$ entonces el efecto parece el resultado o bien de una coincidencia o bien el producto de una fuerza misteriosa presente en todo el conjunto de la esfera. La nueva energía que ha entrado en juego es imposible de localizar y su figura es la de un azar o la de una inmanencia" ${ }^{31}$.

Ahora bien, el negar la aprehensibilidad empírica del acto creador, inmergiéndolo en los determinismos de las causas segundas, no implica el que se nieguen otras formas de aprehensibilidad y, mucho menos, la negación de su existencia. Ni la existencia de antecedentes para cada nueva aparición significa que ellos sean la causa única y adecuada de la misma.

Para Teilhard, es evidente que "en el movimiento ascendente de la vida, se oculta la acción continua de un ser que sostiene y eleva, desde dentro, el universo" " 22 . Dios trabaja en la Vida. La ayuda,

30. Les fondements et le fond de l'idée d'évolution: (Oeu. III, 188).

31. Note sur les modes de l'action divine dans l'univers: (Oeu. $\mathrm{X}, 36$ ).

32. Note sur les modes de l'action divine dans l'univers: (Oeu. $\mathbf{X}, 39$ ). 
la eleva, la da el impulso que la lanza hacia adelante, el apetito que la atrae, el crecimiento que la transforma" ${ }^{33}$ :

\section{La transformación creadora}

En un universo fijista, los teólogos solamente necesitaban las nociones de "creación" y "conservación" para pensar y presentar a nivel teórico las relaciones del Ser Trascendente con el ser participado. La definición clásica de creación ("productio rei ex nihilo sui et subjecti") quiere decir que nada absolutamente preexiste al acto creador: ni la cosa según su perfección formal, ni un punto de apoyo cualquiera a partir del cual las cosas serían hechas. Antes de la creación no existe más que el Ser Necesario. La producción creadora de nuestro universo habia tenido lugar una sola vez y para mantenerlo en el ser hubiese bastado, en el futuro, la divina conservación.

En un universo evolutivo que se va haciendo y perfeccionando, la actividad creadora en cuanto tal no se interrumpe y viene a coincidir, por su extensión, con lo que tradicionalmente se llamaba conservación. En una tal concepción del universo, el instrumental teórico clásico sería insuficiente para la nueva situación. Se impone, según Teilhard, el recurso a una nueva noción: la de "transformación creadora".

La transformación, en la filosofía tradicional, era definida como una "eductio rei ex nihilo sui et potentia subjecti". Con esta definición se quería indicar la substitución de una forma por otra en la materia prima: en estas operaciones no hay, en sentido absoluto, aparición de novedad ontológica, sino una simple substitución. Las formas se intercambian en la materia prima. Para este tipo de operaciones bastan, como explicación, los recursos eficientes propios de la actividad de las causas segundas. Entre la transformación y la creación estricta no habría término medio.

La transformación creadora que Teilhard postula seria una tercera especie de movimiento óntico, entre la creación y la transformación. $Y$ es que, en un universo cuya creación continúa realizándose ante nuestros ojos y en el que la acción creadora se sirve de lo ya creado como precedente de ulteriores creaciones, nos encontramos con cambios que no son ni creaciones ni transformaciones en sentido clásico. A lo largo de la línea del tiempo hay, en efecto, actividad creadora en sentido estricto, en cuanto que en determina-

33. La vie cosmique (ETG 50). 
dos puntos de la misma hay, sin lugar a dudas, aparición de radical novedad y aumento óntico absoluto con relación a los precedentes (v. gr. aparición de la conciencia reflexiva). Pero en esos puntos la actividad creadora no se ejerce sobre la nada absoluta, sino que usa toda la evolución anterior como precedente y punto de apoyo.

Consiguientemente, encontramos un acto en el que se aúnan dos nociones aparentemente irreductibles en los esquemas teóricos clásicos: la creación y la transformación: creación, en cuanto que se da aparición de algo que supera definitivamente a sus antecedentes cósmicos; transformación, en cuanto que el acto creador usa un antecedente (subjectum) como punto de apoyo. Habría, pues, una "productio rei ex nihilo sui et potentia subjecti": algo creado preexistente seria engrandecido hasta el punto de convertirse en algo radicalmente distinto de lo que era antes ${ }^{34}$.

\section{Creación y causa final}

Para hacer de algún modo comprensible el acto creador, el Génesis se sirve de la imagen del artesano. La tradición filosófica, al tratar de "catalogar" la actividad creadora así descrita, escoge la "eficiencia" en el esquema causal aristotélico, como más próxima al pensamiento bíblico.

Según Teilhard, el influjo de la causa creadora no ha de entenderse tanto como algo que empuja la evolución "desde detrás", como la atracción ejercida por un centro de convergencia hacia el que tiende el movimiento del universo ${ }^{35}$. Teilhard denomina "Punto Omega" en sus escritos fenomenológicos y "Cristo Universal" en sus ensayos filosófico-teológicos a este centro de convergencia, motor "ab ante" de la evolución. La atracción de Omega produce una progresiva unificación en el cosmos, es decir, un perfeccionamiento en el ser ${ }^{36}$.

Salgamos al paso de algunas posibles falsas interpretaciones de Omega. Omega no es el producto de la evolución, sino que la pre-

34. Sur la notion de transformation créatrice: (Oeu. $\mathbf{x}, 29$ ).

35. "Nous comprenons maintenant que ce mouvement paradoxal est soutenu par un premier moteur en avant. La branche monte, non point supporté par sa base, mais suspendue à l'avenir". La place de l'homme dans l'univers: (Oeu. III, 323).

36. "Au départ, nous ne pouvions y voir (ou nous pouvions n'y voir) qu'un mouvement autonome, spontané, de montée de conscience Maintenant nous découvrons que ce flux est une marée provoquée par l'actioin d'un astre suprême. Si le Multiple s'unifie, c'est finalement parce qu'il est attiré": Esquisse d'une dialectique de l'esprit: (Oeu. VII, 152-153). 
existe y la trasciende, precisamente para ser centro de convergencia supremamente atrayente ${ }^{37}$.

La acción creadora es, pues, concebida como un inmanente influjo de atracción, ejercido por un centro de convergencia trascendente, supremamente amable.

Es evidente que este modo de representarse la actividad creadora no se opone, en principio, al dogma de la creación, ya que lo único que éste postula es la total dependencia óntica de la creatura con relación a la Causa Trascendente, sin especificar el cómo de esta dependencia. En cuanto "imagen" posee tanto valor como la tradicional del "artesano". Es más, para los espíritus modernos, formados en la escuela del evolucionismo, posee, quizá, mayor fuerza explicativa que la tradicional. Sin embargo, no debemos olvidar que se trata de una imagen y que las imágenes nunca pueden explicar totalmente una realidad que se nos da en la fe.

Teilhard pretende hacer de Aristóteles el patrón de quienes se representan la actividad creadora según un modelo de causalidad eficiente ${ }^{38}$. Esta opinión no es históricamente justa, ya que, al final de su vida, cuando Aristóteles se pregunta cómo el motor inmóvil mueve a las esferas, se decide por la causalidad final, única causa que no necesita moverse para mover. El motor inmóvil mueve como amado (ஸ́ épófuavov) ${ }^{39}$. Por consiguiente, Aristóteles está más cerca de Teilhard de lo que éste piensa.

Si intentamos reunir en una sola definición final todas las ideas teilhardianas sobre la creación podria, quizás, valer la siguiente: Infiujo empíricamente inaprensible que se ejerce, simultáneamente, sobre la totalidad espacio-temporal del universo y utilizando los elementos de la evolución precedente. Tal influjo se ejerce, a modo de atracción, desde el interior de las causas segundas, sin confundirse con ellas y es la causa explicativa adecuada del cosmos y de las características de su evolución.

37. "Puisque au moins par une part, la plus centrale de lui-même, il est transcendant (c'est-à-dire indépendant de l'évolution), c'est que, par ce centre de lui-même, il subsiste sur soi, indépendant du Temps et de l'Espace. Ce qui revient à dire, que, pour notre expérience, il se comporte comme un Ultra-Foyer de convergence, non seulement virtuel, mais éminement actuel": Esquisse d'une dialectique de l'esprit: (Oeu, VII, 152-153).

38. "Depuis Aristote, on n'avait guère cessé de construire des modèles de Dieu sur le type d'un premier moteur, extrinseque, agissant à retro. Depuis l'émergence, en notre conscience, du sens évolutif, il ne nous est plus physiquement possible de concevoir, ni d'adorer, autre chose qu'un Dieu, premier moteur. organique ab ante": Le Dieu de l'évolution: (Oeu. X, 288).

39. Metaf. L 7, 1072 b 3. 


\section{Critica y valoración}

¿Qué pensar de estas ideas? Es evidente que muchas de ellas se han convertido en patrimonio común de la mayor parte de los teólogos de nuestros días y que representan un notable esfuerzo por adecuar los esquemas tradicionales a las nuevas dimensiones que el cosmos ha adquirido en los últimos decenios.

Es asimismo evidente que Teilhard no niega la creación, sino que, por el contrario, hace omnipresente el influjo del creador, interiorizándolo en los procesos cósmicos "naturales". Para él, la creación coincide, prácticamente, con lo que en la tradición se denominaba conservación. El influjo creador está presente y opera en cada partícula cósmica, pero en ninguna de ellas es aprehensible en cuanto tal, ya que parece confundirse con las actividades propias de la creaturas.

La gran ventaja de esta presentación de la actividad creadora, es su compatibilidad perfecta con la imagen del mundo y de su historia que nos presentan las actuales ciencias positivas: un proceso orgánicamente ligado en el que, al menos a nivel de las apariencias, todo es condicionado en su aparecer por lo precedente. La causalidad propia de la creatura es puesta de manifiesto y se revaloriza la actividad de las causas segundas. Para Teilhard, esta colaboración de las causas segundas en nada merma la grandeza del creador, sino que, por el contrario, es la manera más noble de representarse su actividad, tanto para El como para la creatura. Dios, más que hacer las cosas, hace que éstas se hagan.

Se trata, por consiguiente, de una representación teórica bastante aceptable para quien piense el mundo en esquemas evolucionistas y es perfectamente compatible con la fe en el dogma de la creación.

Desgraciadamente, Teilhard no se conformó con las tesis expuestas, sino que, como en casi toda su obra, hay una posterior elaboración que tiende a universalizar y absolutizar sus teorias. Teilhard, en el fondo, tiene espíritu hegeliano, pues nunca se conforma con situaciones de hecho. A él no le basta constatar cómo son las cosas, sino que necesita descubrir una ley universal que le permita afirmar que son asi y que no pueden ser de otra manera. Tiende irresistiblemente a hacer de todo lo factual una situación de derecho. $\mathrm{Y}$ en el caso presente, no se conforma con presentarnos sus ideas sobre el cómo de la creación, sino que pretende hacer de su modelo el único posible. No se resigna a decirnos cómo las cosas se llevan a cabo, sino que además quiere demostrarnos que otro modo 
de realización es imposible y por qué. Así, por ejemplo, cuando nos dice que Dios utiliza la colaboración de las actividades propias de las causas segundas cuando crea en la evolución, pretende demostrarnos, a continuación, que las causas segundas no pueden no colaborar y que Dios no puede crear de otra manera ${ }^{40}$. Y si nunca crea seres aislados, es porque no puede crearlos sino en el contexto de una evolución ${ }^{41}$ Teilhard ha pretendido reaccionar contra la arbitrariedad manifestada por ciertos teólogos con su constante recurso a la libertad y omnipotencia divinas, pero él acaba por poner tal serie de condiciones a la actividad divina ad extra, que no se ve cómo se salvan ninguna de ellas. El parece haber encontrado las normas ineludibles a que debe someterse el creador en su actividad, caso que pretenda crear. Es más, como veremos, llega a hacer incluso del hecho de la creación una operación casi necesaria.

Esta tendencia a la absolutización es la consecuencia de uno de los rasgos primitivos más fundamentales del "hombre" Teilhard. El mismo reconoce que, en él, hay inclinaciones naturales irreformables ${ }^{42}$. $\mathrm{Y}$ entre ellas menciona su preferencia por lo necesario ${ }^{43}$. Esta pasión fundamental e irreformable le lleva, de hecho, en numerosas ocasiones a absolutizar y universalizar situaciones de hecho, convirtiéndolas en leyes generales.

Sus ideas sobre la creación son presentadas al final de su vida con una tal falta de flexibilidad que parecen incompatibles con los atributos fundamentales del ser supremo ${ }^{* 4}$. Así, la creación se convierte en una operación necesaria, no sólo en cuanto al modo de su realización, sino en cuanto al mismo hecho. Ya en algunos escritos de su juventud se sintió atraido por la idea de hacer de la creación

40. "Nous avons déjà reconnu une première loi très générale à laquelle est soumise l'opération divine ad extra: celle de ne pouvoir agir (en vertu même de sa perfection) en rupture des natures individuelles ou en dysharmonie avec la marche de l'ensemble - c'est-à- dire sur un même plan que les causes secondes": Note sur les modes de l'action divine dans l'univers: (Oeu. $\mathrm{X}, 42$ ).

41. Christologie et évolution: (Oeu. $\mathrm{X}, 101)$; Note sur les modes de l'action divine dans l'univers: (Oeu. $\mathrm{X}, 42$ ).

42. Mon univers (1918): (ETG 267).

43. Le coeur de la matière (inédito), p. 4.

44. Sobre todo en Comment je vois (1948): (Oeu. XI, 177-224.

45. Nos estamos refiriendo a las ideas expuestas en "La lutte contre la Multitude" (ETG 109-132) y en "L'union créatrice" (ETG 169-197). En ambas obras parece tener una concepción maniquea o emanantista de la creación inicial. Dios no partiría de la nada absoluta para realizar su obra, sino que, desde siempre, a la unidad substancial divina se opondría, como un desafío y una súplica, lo que él llama "múltiple inicial" y "nada física". La "nada física" seria el punto de apoyo de la creación que consistiría en una unión progresiva de la misma. A 
una operación divina inevitable ${ }^{45}$, pero al final de sus días ya no se trata simplemente de un deseo, sino de una tesis formulada con todo rigor y claridad. Decir que Dios ha creado el mundo libérrimamente y por amor, es calificado por Teilhard de "respuesta sentimental" ${ }^{46}$. La creación es una operación de absoluto interés para Dios ${ }^{47}$, pues sin ella le faltaría algo fundamental ${ }^{48}$. Teilhard llega hasta el punto de calificar a la creación de "fase de la Teogénesis". Dios se constituiría como en dos fases: una atemporal (Trinitización) y otra temporal (Creación), consistente en la unificación máxima del sistema real universal ${ }^{49}$. Si queremos encontrar la palabra que designe, según Teilhard, la historia del ser y su evolución, deberiamos recurrir a la terminología paulina y hablar de "pleromización". La "pleromización" consistiria en la progresiva victoria de la Unidad divina, sobre la aureola de Multiplicidad primitiva que su misma unidad hizo surgir. Hay, pues, una vuelta a sus teorías juveniles sobre el Múltiple imponderable que él denomina "nada física" 50. La "pleromización" es un proceso necesario e inevitable. Y esto, no sólo porque el Ser Necesario no puede "resistir", al parecer, a la muda súplica del Múltiple que pone en su periferia ${ }^{51}$, sino porque de su resultado va a depender su plenitud. El proceso es lento y trabajoso y el Mal es un subproducto inevitable. Si Dios quiere

pesar de la confusión verbal y de los intentos de hacer de la "nada física" una verdadera nada compatible con el dogma de la creación, hemos de decir que esta nada física es, efectivamente, algo, pues la nada absoluta ni suplica ni desafía. La creación es descrita en el estilo de una epopeya metafísica en la que la Unidad Suprema, en lucha con la dispersión y multiplicidad iniciales, va creando el mundo a través de unificaciones sucesivas.

46. L'âme du monde: (ETG 231).

47. L'unión créatrice: (ETG 184-185); Cahier 3; (inédito); 4 noviembre 1917.

48. Christianisme et évolution: (Oeu. $\mathrm{X}, 213$ ).

49. "On pourrait dire que, pour notre raison discursive, tout se passe comme s'il y avait deux phases dans la Théogènese. Au cours de la première phase, Dieu se pose dans sa structure trinitaire (l'être fontal se réfléchissant, selfsuffissant, sur lui-même): Trinisation. Au cours de la deuxième phase, Il s'eveloppe de l'être participé, par unification évolutive du Multiple pur (Néant positif) né, à l'état de potentialité absolue, par antithèse à l'unité trinitaire une fois posée: "Création". Christianisme et évolution: (Oeu. X, 208-209, nota 1).

50. "Par le fait même qu'il s'unifie sur soi pour exister, l'être premier fait ipso facto jaillir une autre espèce d'opposition, non plus au coeur, mais aux antipodes de lui même... L'unité self-subsistante, au pôle de l'être; et nécessairement par suite, tout au tour, à la périphérie, le Multiple": Comment je vois: (Oeu. XI, 209).

51. “...une imploration d'être, à laquelle (et c'est ici que notre intelligence ne sait décidément plus, à telles profondeurs, comment distinguer suprême nécessité de suprême liberté), à laquelle, dis-je, tout se passe comme si Dieu n'avait pu résister": Comment je vois: (Oeu. XI, 209). 
crear, sabe que tiene que aceptar la presencia del Mal a lo largo del proceso.

Tampoco la Encarnación ni la Redención son eventos libres ni arbitrarios, sino etapas orgánicamente necesarias en el proceso unificante de "pleromización" 52 . Todos los misterios cristianos tienen una función cósmica necesaria en la consecución del sistema real universal: conseguir el máximo grado de unificación posible ${ }^{53}$.

De 10 expuesto se deduce que no exagerábamos cuando hablabamos del "hegelianismo" teilhardiano. Cuando Hegel, el filósofo de lo universal y de lo absoluto, se aleja definitivamente de Schelling los motivos que aduce son dos: que el principio de su sistema no se demuestra necesariamente, sino que es simplemente puesto y que las diversas etapas del sistema no son deducidas de una manera absolutamente necesaria. Es evidente que Teilhard está animado de un espíritu parecido: él quiere una síntesis de lo real absolutamente última, en la que sus elementos ocupen en ella un lugar natural. Lo fortuito, lo libre y lo arbitrario le ponen nervioso. La consecuencia es la destrucción del carácter sobrenatural y gratuito de los principales misterios cristianos.

Dotadas de este carácter de necesidad y absolutez no pueden ser aceptadas las ideas teilhardianas sobre la creación. Si Teilhard se hubiese conformado con exponernos lo que podrían ser las grandes líneas teóricas de nuestra representación del actuar creador, sus ideas serían bastante aceptables. Querer hacer, sin embargo, de constataciones factuales leyes ineludibles a que debe someterse el Creador es destruir la libertad divina. $Y$ sus últimas tesis, tal como son formuladas, son incompatibles con la autosuficiencia divina.

Uno de los motivos por los que Teilhard encuentra tantas difcultades a la hora de buscar una representación teórica adecuada a ciertos dogmas cristianos es una conformación especial de toda su psicologia, por la que tiene necesidad de representarse "plásticamente" las cosas. Esta tendencia natural de su psicología se vio reforzada por el género de vida que tuvo que llevar. Obligado durante la mayor parte de su vida a pensar y escribir desde una epistemo-

52. Du cosmos à la cosmogènese: (Oeu. VII, 271).

53. "Dans l'enseignement vulgaire, il est couramment admis: 1) que Dieu pouvait absolument (simpliciter) créer ou ne pas créer 2) que s'il créait, il pouvait le faire avec ou sans Incarnation 3) et que s'il s'incarnait, il pouvait le faire péniblement ou non péniblement. C'est ce pluralisme conceptuel qu'il me paraît, en tout hypothèse, essentiel de corriger": Comment je vois: (Oeu. XI, 213, nota 3). "Pas de Dieu (jusqu'à un certain point) sans union créatrice. Pas de création sans immersion incarnatrice. Pas d'Incarnation sans compensation rédemptrice": Comment je vois: (Oeu. XI, 213). 
logía positivista de tipo "cientifista", no puede liberarse totalmente de sus esquemas, ni siquiera cuando se trata de representarse dogmas sobrenaturales. Todo ello se vio además agravado por la tendencia que tiene Teilhard a pensar todo en categorías evolutivas. Ahora bien, lo típico del pensamiento evolutivo es el esquema "antecedente-consecuente": los componentes de la realidad no son descritos como bloques aislados o independientes, sino como momentos o etapas de un flujo ininterrumpido. Cada ser y cada comportamiento es solidario con todo el resto y en sus antecedentes encuentran la explicación de su lugar en el todo.

Todo ello hace que Teilhard necesite ver un "mecanismo" y un proceso solidario para que algo le resulte aceptable y comprensible. Decir solamente que Dios ha creado el mundo o que se ha encarnado, sin explicar el "mecanismo" ni los "antecedentes" de estos eventos, le parecen a Teilhard explicaciones puramente verbales que nada resuelven. Es necesario que tales acontecimientos tengan "antecedentes" que expliquen su por qué y que los doten de un lugar natural en el devenir total. $Y$ este es precisamente su error de perspectiva: considerar acontecimientos "normales" a lo que son misterios sobrenaturales y pretender representárselos dentro de esquemas físicos "normales".

Hay, además, una objeción fundamental a todo el proyecto teilhardiano. $Y$ es que si sus teorias son bastante aceptables cuando describen el actuar creador en la evolución, no lo son tanto cuanto se trata de explicar el origen del universo. Una cosa es explicar la aparición de nuevos seres a lo largo de la historia del mundo y otra, muy distinta, hablarnos del comienzo de lo finito. $Y$, en este punto, Teilhard nos propone teorias bastante peregrinas. Ya dejamos consignado en una nota el proyecto teilhardiano de extender a la creación inicial los mecanismos de unión que aplica a la evolución del mundo. La "nada" de que nos habla, reflejo de la Unidad Suprema, es en definitiva, "algo". $Y$ entonces sus relatos adquieren todos los caracteres de un maniqueísmo real, por más que las palabras pretendan decir otra cosa.

Es, sin embargo, indudable que, incluso en sus tesis erróneas, su obra ha significado un poderoso impulso en orden a la renovación de multitud de ideas que se consideraban como definitivas. En especial, ha promovido el reajuste de la Teología al nuevo marco de un mundo en evolución. Sus doctrinas han sido, al menos, punto de partida para nuevas reflexiones sobre el problema del mal, del pecado original, del valor de las actividades, de las causas segundas, de las funciones cósmicas de Cristo, etc... Y, desde luego, él nunca 
consideró sus teorias, en contra de lo que hacen algunos de sus seguidores, como absolutamente ciertas y definitivas.

\section{Teilhard sobre el "teilhardismo"}

Como conclusión de nuestro trabajo no podemos por menos de exponer la opinión de Teilhard sobre sus ideas. Este detalle nos mostrará una grandeza de alma poco común y la maravillosa amplitud de espíritu de un gran pensador.

Teilhard no propone sus ideas de manera presuntuosa o como si se tratase de algo acabado y que no admite discusión, sino como ensayo de clarificación del propio pensamiento o como bases de una discusión con quienes tienen el derecho y la obligación de guiarle ${ }^{54}$. El es consciente de la "torpeza y aproximación de los términos" 55 , del valor meramente aproximativo de sus exposiciones ${ }^{56}$, que, a veces, presentan verdaderas y graves objeciones ${ }^{57}$, de proponer tesis paradójicas en las cuales abundan obscuridades y extrañezas ${ }^{58}$, de exponer "a título provisorio, una mezcla de verdad y de error" ${ }^{59}$. Todo ello, para que una expresión más ortodoxa no aniquile en sus ideas una posible "dirección de verdad" ${ }^{00}$ en la que basar ulteriores construcciones del pensamiento. A veces redacta sus ensayos en la forma que lo hace "por no poder hacerlo mejor y provisoriamente" ${ }^{61}$. Reconoce sin pena que sus teorias sobre la unión creatriz tienen necesidad de correcciones, al menos por lo que a la creación inicial se refiere ${ }^{62}$.

La prueba más evidente de esta actitud mental de apertura es que él nunca rehuyó el diálogo o la discusión, sino que redacta una y otra vez un mismo ensayo para proyectar un poco más de luz sobre los problemas en litigio.

El mismo dijo una vez que su mejor seguidor sería aquel que comenzase donde él había terminado y que comprenderle perfectamente significaba ir más allá de donde él mismo fue.

54. "Pour me préciser à moi-même ma propre doctrine et faciliter à ceux qui ont le droit de me guider leur tâche de critique et de redressement, j'ai donc cherché à définir les caractères primitifs et essentiels de ma vision du monde": Mon univers (1918): (ETG 267).

55. Mon univers (1924): (Oeu. IX, 66).

56. Lettre du 24 mars 1917: (Génèse d'une pensée 245). Sobre esta colención de cartas véase la bibliografía de Teilhard al final.

57. L'unión créatrice: (ETG 185).

58. L'union créatrice: (ETG 175).

59. L' union créatrice: (ETG 176).

60. Lettre du 24 mars 1917: (Génèse d'une pensée 245).

61. Mon univers (1918): (ETG 267).

62. Mon univers (1918): (ETG 277). 
Si su postura intelectual se endurece un tanto al final de su vida, ello es bastante comprensible, dadas las circunstancias externas en que, durante toda su vida, se ve obligado a filosofar: "Desterrado", practicamente desde el comienzo de su vida intelectual, vive en medio de un ambiente científico y naturalista y se ve privado de confrontar sus ideas con filósofos y teólogos de profesión ${ }^{63}$.

Si Teilhard vio con gran claridad la necesidad de "actualizar" ciertas teorias tradicionales, para hacerlas compatibles con la imagen científica del mundo en que le tocaba vivir, y en la mayor parte de sus críticas puso verdaderamente el dedo en la herida, no se puede decir que las soluciones que él propuso fuesen siempre muy afortunadas. Pero es innegable el mérito de haber llamado la atención sobre los verdaderos datos del problema.

El siempre se sometió, por otra parte, a la autoridad de sus superiores. Quien conozca un poco su correspondencia y sus relaciones en el mundo científico sabrá la frecuencia y el ahinco con que fue invitado a abandonar su orden para poder llevar una vida intelectual "normal" y poder dar publicidad a sus ideas. Sin embargo, él siempre permaneció fiel a sus convicciones religiosas, sacrificando a ellas su éxito personal.

El juicio teilhardiano sobre el valor del propio esfuerzo intelectual se resume adecuadamente en la siguiente frase:

"Y si esta segunda manera de pensar (la metafísica de la unión) no llega todavía a justificar en el creyente la legítima necesidad, que es su vida, de aportar con su esfuerzo vital algo irreemplazable para el mismo Dios, no nos desanimemos y busquemos todavia mejor" "64.

MaRCELIANo ARRANZ, OSA. Universidad Pontificia

SALAMANCA

63. Así escribe Lubac sobre el aislamiento intelectual de Teilhard de Chardin: “...ses Supérieurs, presque tous n'eurent qu'une idée: le proteger par des mesures de prudence. Il le savait, et il leur en savait gré. Mais ces mesures, qui en fin de compte favorisèrent peut-être plus sa carrière scientifique qu'elles ne la gênèrent, devaient produire d'autre part un résultat contraire à celui que l'on escomptait... Teilhard poursuivit une réflexion trop solitaire. Si précieux à plus d'une égard que soient les fruits de cette réflexion, on ne peut s'empêcher de constater avec mélancolie ce que parfois la systématisation de la pensée teilhardienne en sa dernière phase offre d'étroit et de durci": Pierre Teilhard de Chardin. Lettres intimes à Auguste Valensin, Bruno de Solages, Henri de Lubac (19191955) (Paris 1972) 9.

64. Contingence de l'univers et goût humain de survivre: (Oeu. $\mathrm{x}, 272$ ). 


\section{NOTA BIBLIOGRAFICA.}

1. Sobre las razones seminales.

SCHEPENS, P., "Num S. Augustinus patrocinatur evolutionismus": Gregorianum 6 (1925) 216-230.

HUFFER, E., "Augustinus en de evolutieleer": Studien 113 (1930) 353-368.

SINETY, R. de., "Saint Augustin et le transformisme": Archives de Philosophie 7 (1930) 244-272; 496-524.

FERNANDEZ, A., "La evolución cosmogónica y biológica según S. Agustín": Religión y cultura 15 (1931) 215-237.

BOYER, Ch., "La theorie augustinienne des raisons séminales": Miscellanea agostiniana II (Roma 1931) 795-819.

PERA, L., La creazione simultanea e virtuale secondo S. Agostino. L'atomo virtuale. Firenze, 1934.

NAAME, D., L'idée directrice. L'évolutionisme dans S. Augustin. Paris, 1934.

IBERO, J. M., "Las razones seminales en S. Agustín y los genes biológicos": Comillas (Miscelanea) I (1943) 527-557.

ARTHURS, J., "Saint Augustin et l'évolutionisme": Revue de l'université Laval 2 (1947-1948) 89-95.

CAPDET, R., "Les raisons séminales d'après St. Augustin": Bulletin de littérature ecclésiastique 50 (1949) 208-228.

CILlERUELO, L., "La formación del cuerpo según S. Agustín": La Ciudad de Dios 66 (1950) 445-473.

CHRISTIAN, W. A., "Augustine on the creation of the world": The Harvard Theological Review 46 (1953) 1-25.

THONNARD, F. J., "Les raisons séminales selon S. Augustin": Actes $d u \quad X I$ Congrès International de Philosophie (1953), XII 146-152.

MITTERER, A., Die Entwicklungslehre Augustins im Vergleich mit dem Weltbild des hl. Thomas von Aquin und dem Gegenwart. Wien/Freiburg 1956.

HOLL, A., Seminalis ratio. Wien, 1961.

BRADY, J. M., "St. Augustine's Theory of Seminal Reasons": The New Scholasticism 38 (1964) 141-158.

2. Sobre Teilhard y la creación

a) La Bibliografía en torno a Teilhard es inmensa. De los muchos intentos de compilación que han aparecido el más completo es el de Ladislaus POLGAR, que en el Archivum historicum Societatis Jesu (Fascículo 2, parte VI de la sección "Bibliographia de Historia Societatis Jesu") publica un resumen anual de la literatura en torno a Teilhard. Hasta ahora han aparecido resúmenes desde 1955 hasta 1976.

b) La obra teilhardiana

Oeuvres de Pierre Teilhard de Chardin I-XI. (Paris-Seuil), 1955-1973.

Ecrits du temps de la guerre (1916-1919). Paris, 1965.

$L e$, coeur de la matière. Autobiografía intelectual inédita. Parcialmente publicada en Europe 43 (Paris 1965) 105-114.

Lettres d'Egypte (1905-1908). Paris, 1963.

Lettres d'Hastings et de Paris (1908-1914). Paris, 1965.

Génèse d'une pensée: Lettres 1914-1919. Paris, 1961.

Lettres de voyage (1923-1955). Paris, 1961.

Lettres à Léontine Zanta. Paris, 1965.

Accomplir l'homme. Lettres inédites. (1926-1952). Paris, 1968.

Dans le sillage des Sinanthropes. Lettres inédites de Pierre Teilhard de Chardin et de Johan Gunnar Anderson (1926-1934). Paris, 1971. 
Pierre Teilhard de Chardin. Lettres intimes à Auguste Valensin, Bruno de Solages, Henri de Lubac (1919-1955). Paris, 1972.

c) Algunos trabajos valorativos importantes sobre el Teilhardismo

ABASCAL COBO, M. A., Cosmología evolutiva. Santander, 1974.

BARTHELEMY-MADAULE, M., Bergson et Teilhard de Chardin. Paris, 1963.

CUENOT, C., Pierre Teilhard de Chardin. Les grandes étapes de son évolution. Paris, 1958.

DELFGAAUW, B., Teilhard de Chardin. Baarn, 1961.

LUBAC, H. de, La pensée religieuse du Père Pierre Teilhard de Chardin. Paris, 1962.

LUBAC, H. de, La prière du Père Teilhard de Chardin. Paris, 1964.

LUYTEN, N. A., Teilhard de Chardin. Nouvelles perspectives du savoir? Fribourg, 1965.

LUYTEN, N. A., "La méthode du Père Teilhard": Teilhard de Chardin et la pensée catholique (Colloque de Venise sous les auspices de Pax Romana), (Paris 1965), 19-27.

LUYTEN, N. A., "Materie-Bewusstsein-Geist, in der Sicht Teilhard de Chardins"; Teilhard de Chardin. Philosophische und theologische Probleme seines Denkens, (Würzburg 1967), 53-87.

LUYTEN, N. A., "Die Materie, Quelle des Geistes? Das Entstehen des Geistes in der Evolution", en Teilhard de Chardin und das Problem des Weltbilddenkens, (Freiburg/München 1968), 117-140.

ORMEA, F., Teilhard de Chardin. Guida al pensiero scientifico et religioso (2 v.) Firenze, 1968

RABUT, O. A., Dialogue avec Teilhard de Chardin. Paris, 1968.

RIDEAU, E., La pensée du Père Teilhard de Chardin. Paris, 1965.

SOLAGES, B. de, Teilhard de Chardin. Témoignage et étude sur le développement de sa pensée. Toulouse, 1967.

SMULDERS, P., La visión de Teilhard de Chardin. Essai de réflexion théologique. Paris/Bruges, $1964 . \ldots . .$.

TRESMONTANT, C., Introduction à la pensée de Teilhard de Chardin. Paris, 1956.

VIALLET, F. A., Teilhard de Chardin. Zwischen Alpha und Omega. Das Weltbild Teilhard de Chardins. Nürnberg/Zürich, 1963.

VIALLET, F. A., Teilhard de Chardin. Zwischen Ja und Nein. Dialog, Dokumente, Kritik. Nürnberg/ Zürich, 1963.

d) El problema de la creación en Teilhard

ALTNER, G., Schöpfungsglaube und Entwicklungsgedanke in der protestantischen Theologie awischen Ernst Haeckel und Teilhard de Chardin. Zürich, 1965.

BLANCHARD, J. P., "Imploration d'être et création": Univers 2 (1965) 59-69.

CHAUCHARD, P., "Evolutionnisme matérialiste et fixisme ontologique dans la création évolutive": Bulletin de la société française de philosophie (n. spé.) 1959.

COLOMER, E., "Evolutió, creació y providència": Questions de vida cristiana 47 (1969) 57-82.

CORVEZ, M., "Création et évolution du monde": Revue thomiste 64 (1964) 549568.

TOLLENAERE, M. de, "Weltschöpfung und Entwicklung. Teilhard de Chardins. Visión und das christliche Dogma": Wort und Wahrheit 16 (1961) 273-282.

ELLIOT, F. G., "The creative aspect of evolution": International Philosophical Quarterly 6 (1966) 230-247.

GRAY, D. P., Creative Union in Christ in the Thought of Theilhard de Chardin. New York, 1968. 
HAAS, A., "Schöpfungslehre als Physik und Metaphysik des Einen und Vielen bei Teilhard de Chardin. Nach der unveröffentlichten Schrift Comment je vois": Scholastik 39 (1964) 321-342; 510527.

HENGSTENBERG, H., Evolution und Schöpfung. Eine Antwort auf den Evolutioismus Teilhard de Chardins. München, 1963.

HRACHOVEC, H., "Teilhard de Chardin und die Theologie der Zukunft. Ein Kapitel im Streit um die Ontologie in Theologie": Wort und Wahrheit 27 (1972) 273-284.

LAMBILLIOTE, M., "Evolution et création": Synthèses 111 (1955) 345-354.

LAY, R., Der neue Glaube an die Schöpfung. Olten und Freiburg i. Br., 1971.

LOPEZ, S. C., "Metafísica y creación en Teilhard de Chardin": Sapientia 20 (1965) $275-289$

NORTH, R., "Teilhard and the Problem of Creation": Theological Studies 24 (1963) 577-601.

OVERMAN, R. H., Evolution and the Christian Doctrine of Creation. London, 1968.

RABUT, O., Le problème de Dieu inscrit dans l'évolution. Paris, 1963.

SAHAGUN, L. F., J., "La acción creadora vista por Teilhard de Chardin": Verdad y Vida 28 (1970) 493-518.

SAHAGUN, L. F., J., "Teilhard de Chardin y el estatuto del ser": Pensamiento 29 (1973) 73-104.

SMITH, R. B., "God and evolutive creation": Teilhard reassessed. (London 1970), 41-58.

SMULDERS, P., "Evolutieleer en teekomstverwachting bij Teilhard de Chardin": Bijaragen 21 (1960) 233280.

SMULDERS, P., "Schöpfung, Entwicklung und Vollendung. Zum Anliegen Teilhard de Chardins": Wissenschaft und Wahrheit 27 (1964) 12-29.

ZILLES, U., "Criaçāo e evolução": Vozes 60 (1966) 803-817. 